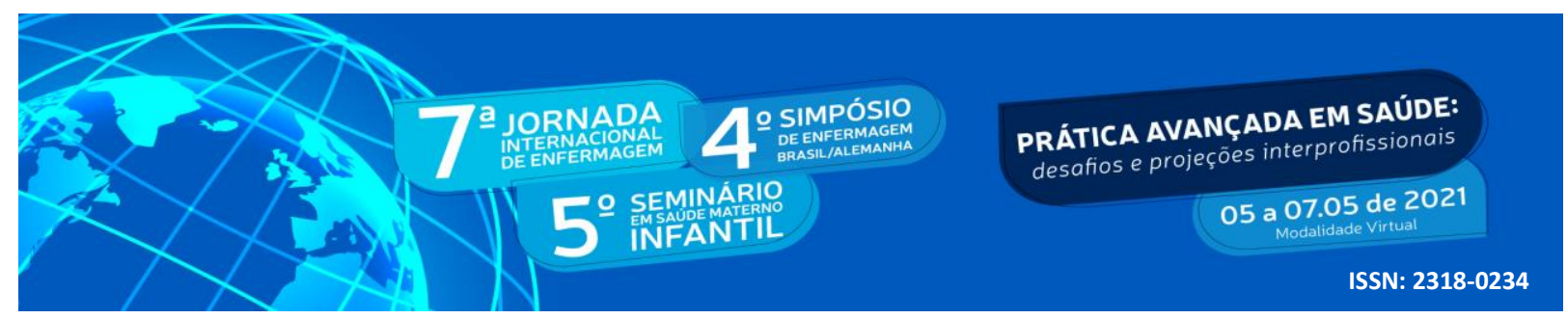

DOI: http://doi.org/10.48195/jie2021-076

\title{
CONTINUUM DO CÂNCER NA REDE DE ATENÇÃO À SAÚDE: O ENSINO NA GRADUAÇÃO EM ENFERMAGEM ${ }^{1}$
}

\section{Clara Beatriz Teixeira Lima Cavalcante ${ }^{2}$; Vanessa de Almeida Ferreira Corrêa ${ }^{3}$; Sonia Regina de $\operatorname{Souza}^{4}$ e Andressa Teoli Nunciaroni ${ }^{5}$}

\section{RESUMO}

Objetivo: Identificar de que forma ocorre a abordagem do Continuum do Câncer na Rede de Atenção à Saúde a partir de discentes de enfermagem durante o curso de graduação. Metodologia: Pesquisa quantitativa, desenvolvida em uma Escola de Enfermagem de uma Universidade Federal, entre novembro de 2020 e fevereiro de 2021, através de questionários virtuais. Participaram 54 acadêmicos de diferentes períodos. Resultados: $\mathrm{O}$ ensino da oncologia durante a graduação compreende a discussão dos estágios do Continuum do Câncer, entretanto, há uma maior ênfase no ponto de atenção hospitalar quando comparada aos cenários ambulatorial e Atenção Primária. Conclusão: A articulação entre o Continuum do Câncer e a rede de atenção potencializa a formação do discente de enfermagem para a inserção no SUS na prevenção e controle do câncer nos diferentes pontos de atenção à saúde.

Palavras-chave: Enfermagem Oncológica; Ensino; Graduação; Oncologia.

\begin{abstract}
Objective: Identify how the Cancer Continuum approach in the Health Care Network occurs from nursing students perspective during the undergraduate course. Methodology: Quantitative research, developed at a Nursing School of a Federal University, between November 2020 and February 2021, through virtual questionnaires. 54 academics from different periods participated. Results: The teaching of oncology during graduation includes the discussion of the stages of the Cancer Continuum, however, there is a greater emphasis on the point of hospital care when compared to the outpatient and primary care settings. Conclusion: The articulation between the Continuum of Cancer

\footnotetext{
${ }^{1}$ Trabalho Original, Iniciação Científica CNPq

2 Discente do Curso de Graduação em Enfermagem da Escola de Enfermagem Alfredo Pinto. Universidade Federal do Estado do Rio de Janeiro UNIRIO. E-mail: clara.beatriz@edu.unirio.br

${ }^{3}$ Orientador. Doutorado em Enfermagem. Escola de Enfermagem Alfredo Pinto. Universidade Federal do Estado do Rio de Janeiro UNIRIO. E-mail: vanessa.correa@unirio.br

${ }^{4}$ Coautor. Doutorado em Enfermagem. Escola de Enfermagem Alfredo Pinto. Universidade Federal do Estado do Rio de Janeiro UNIRIO. E-mail: sonia.souza@unirio.br

${ }^{5}$ Coautor. Doutorado em Ciências da Saúde. Escola de Enfermagem Alfredo Pinto. Universidade Federal do Estado do Rio de Janeiro UNIRIO. E-mail: andressa.nunciaroni@unirio.br
} 


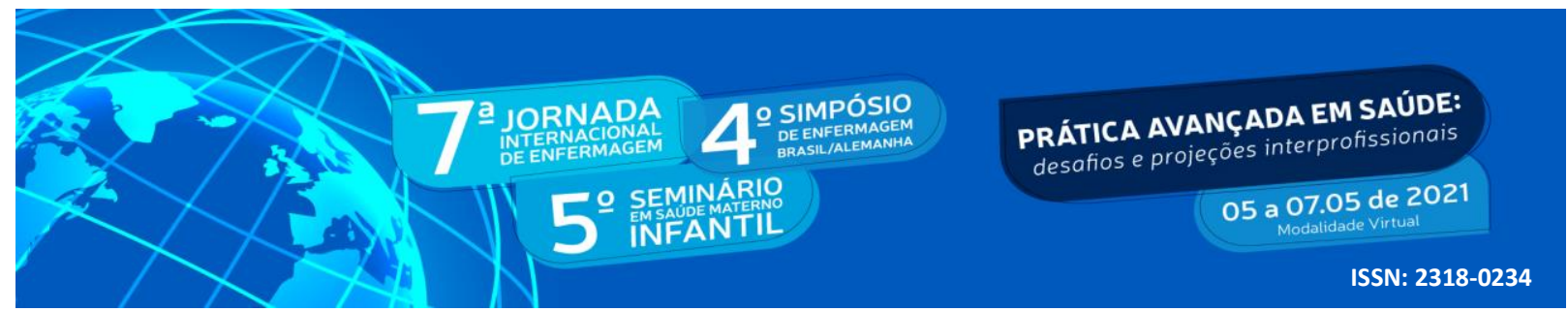

and the care network enhances the training of nursing students for insertion in SUS in the prevention and control of cancer in different points of health care.

Key Words: Teaching; Oncology; Oncology Nursing; University graduate

\section{INTRODUÇÃO}

O câncer configura-se como um grave problema de saúde pública. Entretanto, apesar de ser caracterizado pela sua alta morbimortalidade, intervenções voltadas à promoção da saúde, detecção precoce, diagnóstico e tratamento eficazes são medidas de controle com evidências científicas para reverter o impacto epidemiológico observado ao longo das décadas (INCA, 2020). Sendo necessário caracterizar e refletir quanto a formação em saúde voltada ao ensino da prevenção e controle do câncer na Rede de Atenção à Saúde (RAS).

O National Cancer Institute pertencente à National Institutes of Health conceitua o Continuum do Câncer (Cancer Control Continuum) como os vários estágios de etiologia do câncer, relacionados à: prevenção, detecção precoce, diagnóstico, tratamento, cuidados para a sobrevivência e fim da vida, sendo necessário a formação em saúde e produção de práticas de cuidados voltadas à cada estágio (NATIONAL INSTITUTES OF HEALTH, 2020).

No Brasil, práticas voltadas aos estágios do Continuum do Câncer são organizadas nos diferentes pontos de atenção da Rede de Atenção à Saúde (RAS). Considera-se a RAS, como arranjos organizativos de ações e serviços de saúde, de diferentes densidades tecnológicas, que integradas por meio de sistemas de apoio técnico, logístico e de gestão, buscam garantir a integralidade do cuidado, com provisão de atenção contínua, integral, de qualidade, responsável e humanizada (BRASIL, 2010). Entende-se que, a articulação prática e teórica entre o Continuum do Câncer e a RAS, dialoga com a oferta e o acesso das pessoas ao cuidado de qualidade, desde a prevenção até os cuidados de fim da vida.

Neste contexto, destaca-se o papel do enfermeiro, na atuação em equipe interdisciplinar. O enfermeiro possui responsabilidades na produção do cuidado voltado para todos os estágios do Continuum do Câncer, sendo importante pensar na formação em saúde deste profissional, estratégias para inserir e mobilizar o conhecimento dos discentes quanto aos estágios do 


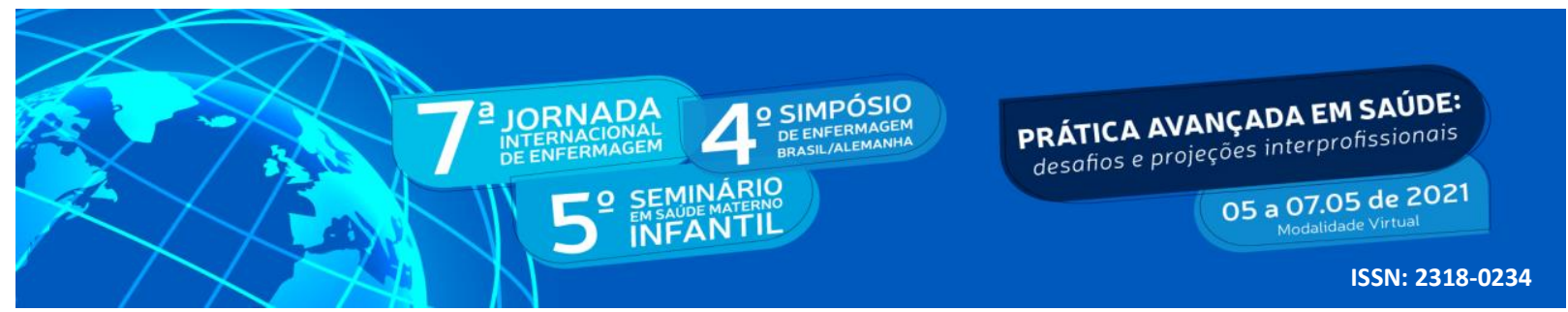

Continuum do Câncer na RAS, ao considerar a pessoa em seu território de vida e as necessidades em saúde por elas apresentadas.

\section{OBJETIVO}

Identificar de que forma ocorre a abordagem do Continuum do Câncer na Rede de Atenção à Saúde a partir de discentes de enfermagem durante o curso de graduação.

\section{METODOLOGIA}

Este artigo é um recorte da pesquisa de Iniciação Científica intitulada "Formação para a prática de cuidado em oncologia sob a ótica de discentes de Enfermagem", trata-se de uma pesquisa com abordagem quantitativa, desenvolvida em uma Escola de Enfermagem de uma Universidade Federal localizada no Estado do Rio de Janeiro (RJ), Brasil. Os critérios de inclusão elencados para os participantes foram: discentes matriculados no Curso de Graduação em Enfermagem da referida instituição cursando a partir do $5^{\circ}$ período; maiores de 18 anos e estarem de acordo com o Termo de Consentimento Livre e Esclarecido (TCLE). Os critérios de exclusão foram: discentes que estejam cursando entre o $1^{\circ}$ e $4^{\circ}$ período e que não aceitem a assinatura do TCLE.

A coleta de dados desenvolveu-se durante os meses de novembro de 2020 a fevereiro de 2021, através de questionários elaborados virtualmente pela plataforma Google Forms. Os participantes foram convidados a participar do estudo por meio de uma Carta Convite, sendo disponibilizado aos possíveis participantes o TCLE para o aceite ou recusa. Responderam ao questionário 54 acadêmicos de enfermagem, que representa uma amostra de 34,4\% dos convites realizados.

Para responder ao objetivo proposto neste artigo, utilizou-se as informações contidas em duas perguntas fechadas, com opções múltiplas de respostas, a saber: "A abordagem do tema (câncer) era focada em quais momentos de desenvolvimento do Câncer?" e "Qual campo da Rede de Atenção à Saúde foi abordado durante esse ensino de oncologia?” Organizou-se os dados em planilhas do Microsoft Excel for Windows e desenvolveu-se análises descritivas. Destaca-se que, essa pesquisa atende a Resolução 466/12 e Resolução 510/16 do Conselho 


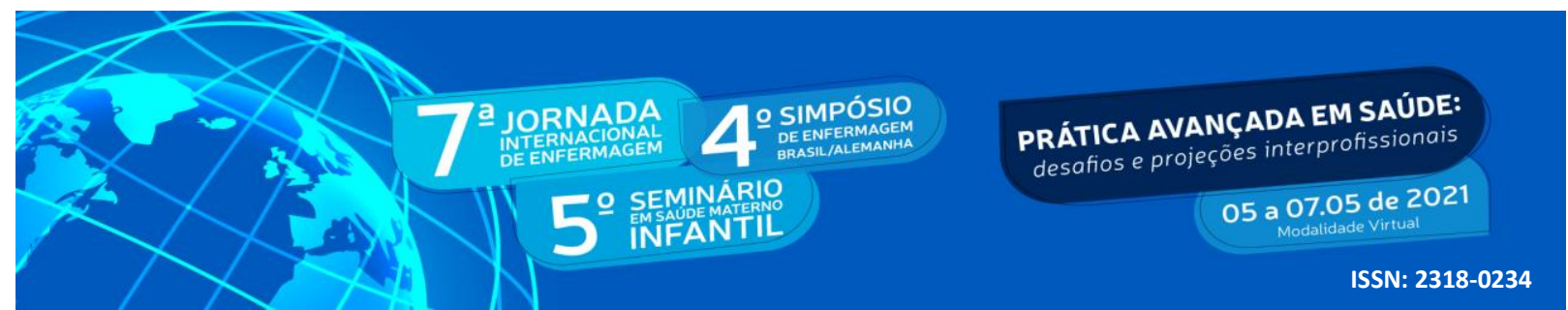

Nacional de Saúde sobre pesquisa com seres humanos, possui aprovação pelo Comitê de Ética em Pesquisa da Universidade Federal do Estado do Rio de Janeiro, sob parecer: 4.214.139 e CAAE: 36196220.8 .0000 .5285 .

\section{RESULTADOS E DISCUSSÃO}

Participaram do estudo 54 discentes do curso de graduação em enfermagem, sendo que 43 deles reportaram o ensino de temáticas ligadas à oncologia. Assim, 43 discentes responderam ao instrumento de coleta de dados relacionado aos estágios do Continuum do câncer e ao campo da RAS onde ocorre as ações de controle do câncer. A caracterização sociodemográfica dos participantes é descrita na Tabela 1.

Tabela 1: Caracterização sociodemográfica dos participantes $(n=54)$

\begin{tabular}{|l|r|r|}
\hline \multicolumn{1}{|c|}{ Variável } & $\mathbf{n}(\%)$ & Média(DP*) \\
\hline Sexo Feminino & $37(68,5)$ & \\
\hline Idade & & $24,3(2,6)$ \\
\hline Período do curso & & \\
$6^{\circ}$ & $13(24,1)$ & \\
$7^{\circ}$ & $15(27,8)$ & \\
$8^{\circ}$ & $16(29,6)$ & \\
Outros & $10(18,5)$ & \\
\hline Formação em oncologia na graduação & $43(79,6)$ & \\
Sim & $11(20,4)$ & \\
Não & & \\
\hline Formação articulada ao currículo da graduação (n=43) & $13(30,2)$ & \\
Sim & $30(69,8)$ & \\
Não & & \\
\hline Forma do ensino de oncologia & $37(86)$ & \\
Aulas teóricas em disciplinas prevista no currículo & $19(44,2)$ & \\
Aulas práticas em disciplinas prevista no currículo & $23(53,5)$ & \\
Liga acadêmica & & \\
\hline
\end{tabular}




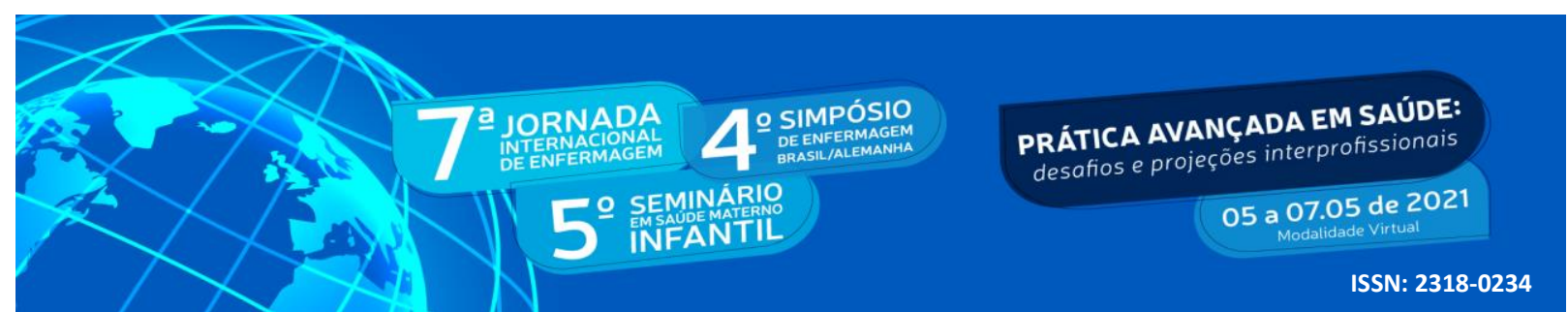

\begin{tabular}{|l|c|c|}
\hline Palestras externas & $\begin{array}{c}26(60,5) \\
6(14)\end{array}$ & \\
Extensão universitária & 6 \\
\hline
\end{tabular}

*DP= desvio padrão

Fonte: Autoria Própria, Rio de Janeiro, RJ, 2021.

No que se refere aos estágios do Continuum do Câncer na RAS abordados durante o curso de graduação, os discentes indicaram com maior frequência a "Detecção Precoce" e “Tratamento". Os resultados da questão 1 são descritos na Tabela 2.

Tabela 2: Estágios do Continuum do Câncer abordados no ensino (n=43).

\begin{tabular}{l|c|c}
\hline Estágio do Continuum do Câncer & $\mathrm{n}$ & Porcentagem \\
\hline Prevenção & 32 & 74,4 \\
Detecção Precoce & 38 & 88,4 \\
Diagnóstico & 29 & 67,4 \\
Tratamento & 37 & 86 \\
Cuidados Paliativos & 34 & 79,1 \\
\hline
\end{tabular}

Fonte: Autoria Própria, Rio de Janeiro, RJ, 2021.

Ressalta-se que, a temática referente aos estágios do câncer é abordada durante o Curso de Graduação em Enfermagem, sendo necessário o ensino dos referidos estágios nos diferentes pontos de atenção. Da mesma forma, é imprescindível identificar se há ênfase no ensino de oncologia em um ponto da RAS em detrimento de outros, de forma a refletir sobre a formação do futuro enfermeiro para o controle do câncer na sua prática profissional. Logo, é necessário que o acadêmico de enfermagem compreenda e se familiarize com os estágios do Continuum do Câncer na RAS, visto que a prevenção e o controle do câncer precisam estar articuladas em todos os pontos de atenção à saúde.

Entende-se que, para o controle do câncer, é necessário desenvolver ações voltadas às estratégias de promoção, prevenção, detecção precoce, tratamento e cuidados paliativos. Além disso, é importante integrar os diversos pontos de atenção: Atenção Primária, Atenção Especializada de Média Complexidade e Atenção Especializada de Alta Complexidade do sistema de saúde (INCA, 2020). Pensar nas formas de produzir práticas em saúde para o controle do câncer, assim como, o acesso dos usuários em toda a RAS, é construir linhas de 


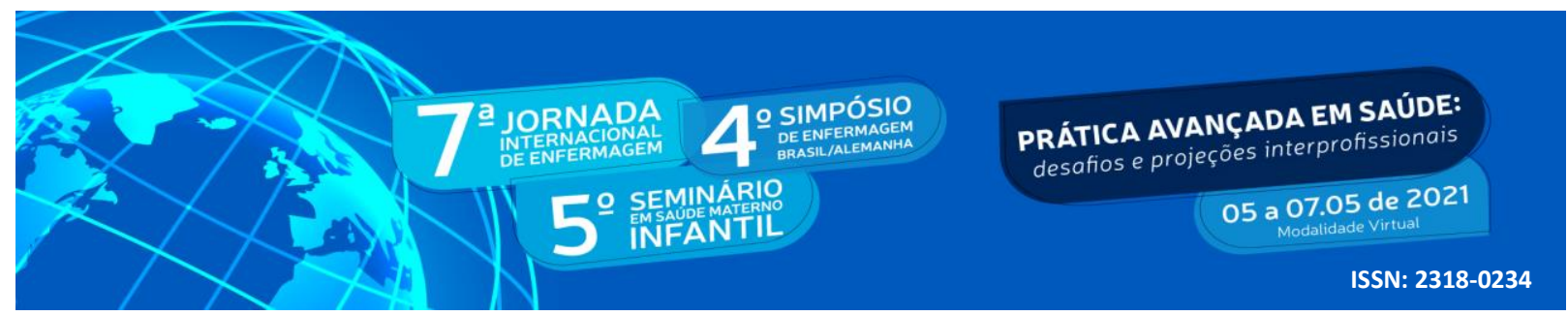

cuidados para apoiar as pessoas em suas escolhas e caminhos, nos serviços e nas ações em saúde.

Assim, organizar o acesso do usuário na RAS e integrar as ações de proteção, promoção, vigilância, prevenção e cuidados à pessoa com câncer é um desafio para os profissionais de saúde e presente nas políticas públicas. Quanto aos estágios do Continuum do Câncer, destacase que as práticas e saberes referentes à prevenção são imprescindíveis. Uma vez que a Organização Mundial da Saúde (OMS) considera que cerca de 40\% das mortes por câncer poderiam ser evitadas, visto que o câncer também é influenciado por fatores externos (INCA, 2020; SILVA, 2016). No que se refere ao estágio de Detecção Precoce, têm-se por objetivo detectar o câncer localizado no órgão de origem, antes de invadir os tecidos circundantes e órgãos distantes, ou detectar uma lesão pré-cancerosa (WHO, 2007). No que tange às estratégias de rastreamento e diagnóstico precoce, objetiva-se diminuir os riscos e possíveis complicações da doença, promovendo o tratamento em tempo oportuno.

Neste continuum necessário para no planejamento das práticas em saúde e no cuidado à saúde da população voltados ao controle do câncer, a abordagem diagnóstica se faz necessária para a educação em saúde referente aos sinais e sintomas, e exames ofertados para obter um diagnóstico e determinar o esquema terapêutico . Quanto ao tratamento, por sua vez, também necessita do ensino na graduação voltado às modalidades de tratamento disponíveis e seu impacto na vida do usuário e família (INCA, 2020). Por fim, a articulação entre os cuidados paliativos e a prática do enfermeiro estão ligados diretamente, pois o profisisonal deverá prestar um cuidado visando a promoção do bem-estar, qualidade de vida e manejo dos sintomas (DE ALMEIDA et al, 2020).

A compreensão da relevância dos diferentes estágios do Continuum do câncer, se faz necessário na formação em saúde, ainda durante o Curso de Graduação em Enfermagem, de modo a aproximar o acadêmico com a prática vivenciada nos diferentes pontos da rede de atenção no Sistema Único de Saúde (SUS).

Para além da reflexão quanto à importância das práticas de controle pautadas nas etapas do Continuum do Câncer, tais práticas devem possuir organização e articular os recursos nos diferentes pontos de atenção, para a garantia do acesso aos serviços de saúde e oferta de um cuidado integral (INCA, 2006). Neste sentido, é importante identificar em quais pontos de atenção à formação em oncologia é abordada. A tabela 3, a seguir, ilustra os resultados 


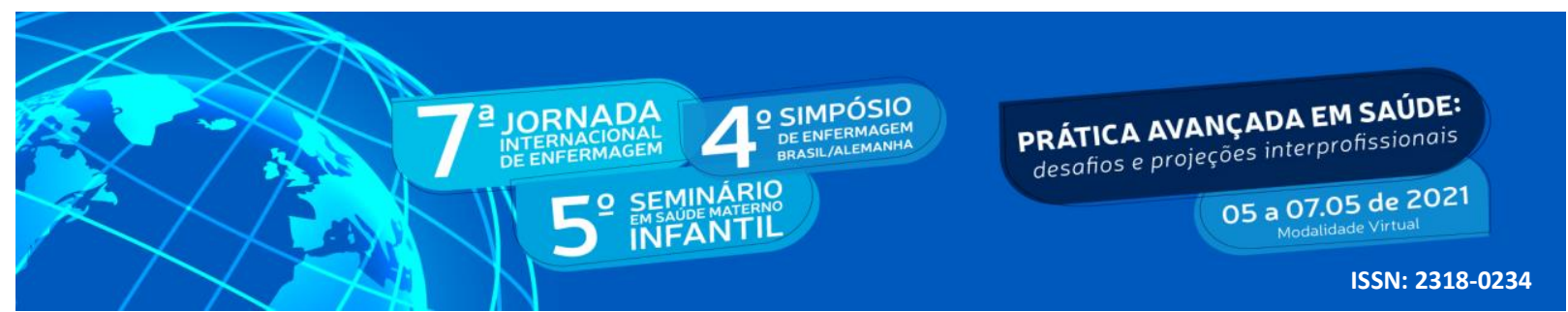

referentes ao campo da Rede de Atenção à Saúde abordado durante esse ensino de oncologia.

Tabela 3: Campo da RAS abordado no ensino ( $n=43)$.

\begin{tabular}{l|c|c}
\hline Estágio do Continuum do Câncer & Frequência & Porcentagem \\
\hline Atenção Primária à Saúde & 22 & $51,2 \%$ \\
Ambulatorial & 24 & $55,8 \%$ \\
Hospitalar & 43 & $100 \%$
\end{tabular}

Fonte: Autoria Própria, Rio de Janeiro, RJ, 2021.

Os resultados encontrados na análise dos questionários evidenciaram que os estágios do Continuum do Câncer na RAS são abordados no ensino da graduação em enfermagem, para além do ensino dos conceitos fisiopatologia oncológica. O ponto de atenção hospitalar foi o cenário de maior articulação no ensino da oncologia, quando comparada às áreas ambulatorial e de Atenção Primária. De acordo com a portaria Portaria $N^{\circ} 874$, a qual institui a Política Nacional para a Prevenção e Controle do Câncer na Rede de Atenção à Saúde das Pessoas com Doenças Crônicas no âmbito do SUS, a assistência integral deve promover atendimento multiprofissional a todos os usuários com câncer, com oferta de cuidado compatível a cada nível de atenção e evolução da doença (BRASIL, 2013).

Para a população sintomática, o primeiro ponto de atenção deve avaliar os sinais e sintomas; e priorizar o encaminhamento para investigação e confirmação diagnóstica no nível secundário. Quando há a confirmação do diagnóstico de câncer, o tratamento é realizado na alta complexidade, em Unidades ou Centros de Assistência de Alta Complexidade em Oncologia (INCA, 2015).

Apesar do ponto de atenção hospitalar ser o cenário que agrega maior experiência dos acadêmicos de enfermagem na formação para o controle do câncer, estudos indicam a importância de pensarmos a atenção primária como um ponto de práticas voltadas ao cuidado no controle do câncer (DE PAULA; VOLOCHKO E FIGUEIREDO, 2016; VIEIRA E TEIXEIRA, 2020)

Segundo o estudo desenvolvido no estado de São Paulo (SP), Brasil, referente aos cuidados à pessoa com câncer de mama e de colo de útero, a linha de cuidados concernente ao 


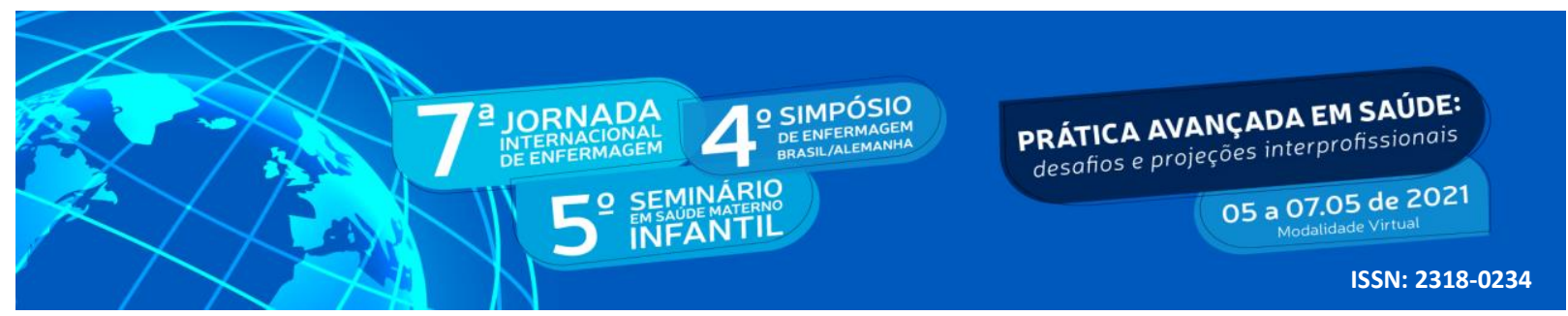

câncer se inicia na atenção primária, com ações de promoção, prevenção e cuidados referentes à identificação precoce e tratamento oportuno. Os autores destacam que, as Unidades Básicas de Saúde (UBS) são porta de entrada do usuário no sistema de saúde, se constituem em espaços importantes de práticas multiprofissionais e possuem uma maior aproximação entre profissional e comunidade (DE PAULA; VOLOCHKO E FIGUEIREDO, 2016).

Assim, ao compreender a relevância da oferta de cuidado compatível a cada nível de atenção e evolução do câncer (BRASIL, 2013), destaca-se a importância da formação do enfermeiro nos cursos de graduação, fundamentados nas ações de controle do câncer nos diferentes pontos de atenção à saúde. Na análise desenvolvida, identificou-se que os pontos da atenção primária, ambulatorial e hospitalar foram reconhecidos como pontos tratados no ensino voltados à formação para a prática do enfermeiro em oncologia no curso de graduação. Porém, apenas 51,2\% dos participantes apontaram a discussão da oncologia na Atenção Primária.

Infere-se que, a menor articulação do ensino em oncologia em um ponto da RAS, pode ocasionar conhecimento ineficaz referente às práticas e serviços ofertados, o que pode impactar na atuação dos profissionais neste ponto da RAS; e na compreensão da Linha de Cuidado voltadas aos estágios do Continuum do câncer. De acordo com Santos; Vieira e Teixeira (2020), a formação do enfermeiro por muitos anos esteve voltada para uma visão centrada no hospital, sem priorizar a saúde coletiva. As influências históricas, ainda nos dias atuais, refletem na profissão enfermagem a dificuldade no processo de transição e adequação do currículo para o docente, discente e usuário do serviço que, por vezes, ainda valoriza o atendimento voltado para a terapêutica hospitalar (SANTOS; VIEIRA e TEIXEIRA, 2020).

Assim, ao articular os estágios do Continuum do câncer e a RAS, a partir das vivências de formação de discentes de enfermagem, refletiu-se sobre a necessidade de ampliação da discussão sobre o controle do câncer nos diversos pontos de atenção da RAS. Apesar da identificação que os estágios Continuum do câncer são abordados durante o Curso de Graduação em Enfermagem, estes devem ser ampliados para a discussão em toda a RAS.

\section{CONCLUSÃO}

O presente estudo possibilitou a discussão de duas abordagens extremamente 


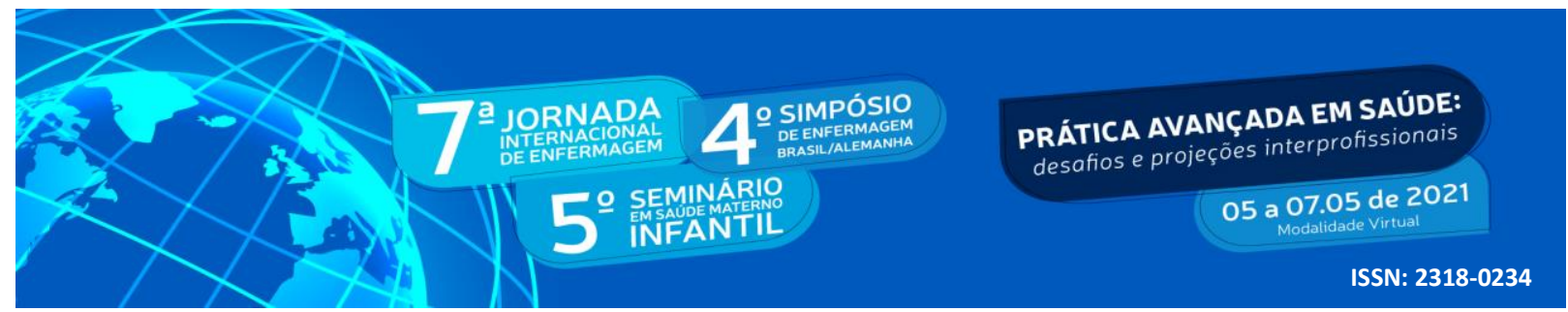

importantes para a formação em saúde nos Cursos de Graduação em Enfermagem e a qualificação das práticas de cuidado em oncologia, tais como: o ensino na Enfermagem para as ações de controle do câncer pautadas nos estágios do Continuum do Câncer e a formação em todos os pontos de atenção à saúde.

A partir das informações coletadas, percebeu-se que o ensino em oncologia durante a graduação compreende a discussão dos estágios do Continuum do Câncer. Tal resultado é relevante no que tange à importância da prática do enfermeiro no Continuum do Câncer, objetivando-se o controle do câncer. Pôde-se observar que, referente aos estágios do Continuum do câncer, desde a promoção e prevenção até os cuidados paliativos são abordados durante a graduação de enfermagem, o que potencializa a formação do discente de enfermagem para a inserção no SUS no controle do câncer em qualquer cenário de atenção à saúde.

Em contrapartida, a análise das respostas dos acadêmicos de enfermagem demonstrou maior vivência dos discentes no ensino no ponto de atenção hospitalar, quando comparado à área ambulatorial e de Atenção Primária. Produzir cuidado no controle do câncer e formas de ensiná-las na prática, a partir do Continuum do Câncer na rede de atenção, a qual organiza o caminho das pessoas e as práticas em cada estágio, torna-se imperativo na formação de enfermeiros frente a este grave problema de saúde pública.

Por fim, salienta-se a importância de novos estudos voltados às estratégias de ensino utilizadas durante o Curso de Graduação em Enfermagem na formação para o cuidado em oncologia. E, assim, aprimorar a formação de novos profissionais da saúde atuantes no SUS, no controle do câncer.

\section{REFERÊNCIAS}

BRASIL. Portaria n. 4.279 de 30 de dezembro de 2010. Estabelece diretrizes para a organização da Rede de Atenção à Saúde no âmbito do Sistema Único de Saúde (SUS). Brasília: Ministério da Saúde, 2010. Disponível em: http://bvsms.saude.gov.br/bvs/saudelegis/gm/2010/prt4279_30_12_2010.html.

BRASIL. Portaria n. 874 de 16 de maio de 2013. Institui a Política Nacional para a Prevenção e Controle do Câncer na Rede de Atenção à Saúde das Pessoas com Doenças Crônicas no âmbito do Sistema Único de Saúde (SUS). Brasília: Ministério da Saúde, 2013. Disponível em: http://bvsms.saude.gov.br/bvs/saudelegis/gm/2013/prt0874_16_05_2013.html. 


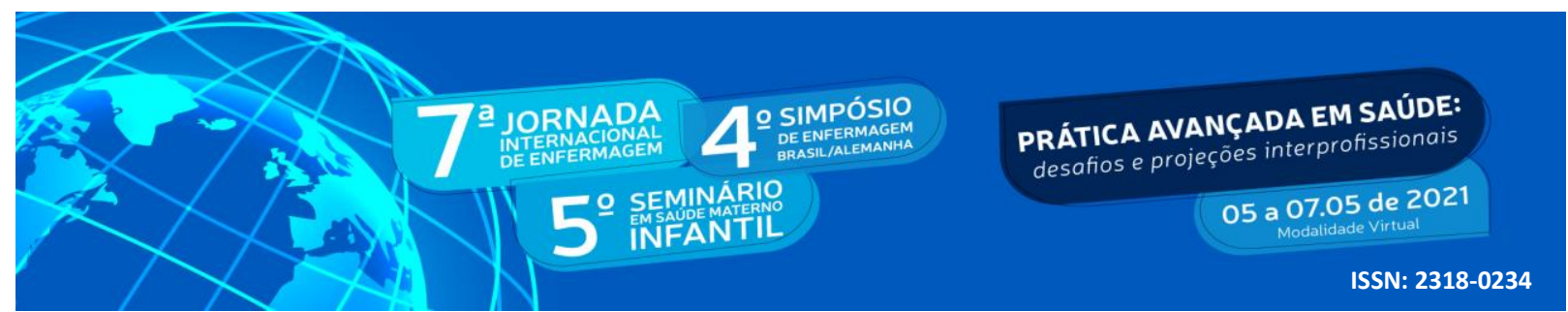

DE PAULA SH.B, VOLOCHKO A, FIGUEIREDO R. Linha de cuidado de câncer de mama e de colo de útero: um estudo sobre referência e contrarreferência em cinco regiões de saúde de São Paulo, Brasil. BIS, Bol. Inst. Saúde. 2016;17(2):146-66.

DE ALMEIDA, P. F. et al. A relação entre o enfermeiro e o paciente nos cuidados paliativos oncológicos. Braz. J. Hea. Rev, Curitiba, v. 3, n. 2, p. 1465-1483, 2020. DOI 10.34119/bjhrv3n2-011.

INSTITUTO NACIONAL DE CÂNCER JOSÉ ALENCAR GOMES DA SILVA - INCA. ABC do câncer: abordagens básicas para o controle do câncer. 6 ed. Rio de Janeiro, 2020.

INSTITUTO NACIONAL DE CÂNCER JOSÉ ALENCAR GOMES DA SILVA . A situação do câncer no Brasil. INCA, Rio de Janeiro, 2006

INSTITUTO NACIONAL DE CÂNCER JOSÉ ALENCAR GOMES DA SILVA. Monitoramento das ações de controle do câncer de mama: Linha de Cuidado e Rede de Atenção ao Câncer de Mama. Rio de Janeiro, 2015.

NATIONAL INSTITUTES OF HEALTH. Nacional Cancer Institute (org.). Cancer Control Continuum. Washington, 2020. Disponível em: https://cancercontrol.cancer.gov/aboutdccps/about-cc/cancer-control-continuum. Acesso em: 15 mar. 2021.

SANTOS, Thadeu Borges Souza; VIEIRA, Silvana Lima; TEIXEIRA, Cristiane Purificação de Oliveira. Rompendo o paradigma hospitalocêntrico na formação de enfermeiro(a)s:

Restruturação de componente curricular técnico-profissionalizante com vistas ao princípio da integralidade. Estudos IAT, Salvador, v.5, n.2, p. 330-349, out. 2020.

SILVA, G. A., et al. The Fraction of Cancer Attributable to Ways of Life, Infections, Occupation, and Environmental Agents in Brazil in 2020. PLoS ONE, 2016 11(2): e0148761.doi:10.1371/journal.pone.0148761

WORLD HEALTH ORGANIZATION (WHO). Cancer control: knowledge into action: WHO guide for effective programmes; module 3. Switzerland, 2007. 